\title{
Status of the HOLMES detector development
}

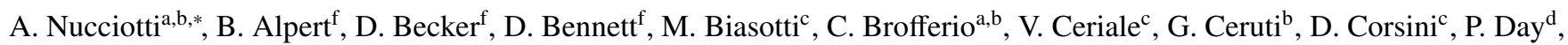
M. De Gerone ${ }^{\mathrm{c}}$, R. Dressler ${ }^{\mathrm{e}}$, M. Faverzani ${ }^{\mathrm{a}, \mathrm{b}}$, E. Ferri ${ }^{\mathrm{a}, \mathrm{b}}$, J. Fowler ${ }^{\mathrm{f}}$, E. Fumagallic ${ }^{\mathrm{c}}$ J. Gard ${ }^{\mathrm{f}}$, F. Gatti ${ }^{\mathrm{c}}$, A. Giachero ${ }^{\mathrm{b}}$,

J. Hays-Wehle ${ }^{\mathrm{f}}$, S. Heinitz ${ }^{\mathrm{e}}$, G. Hilton ${ }^{\mathrm{f}}$, U. Koester ${ }^{\mathrm{g}}$, M. Lusignoli ${ }^{\mathrm{i}}$, M. Maino ${ }^{\mathrm{b}}$, J. Mates ${ }^{\mathrm{f}}$, S. Nisi ${ }^{\mathrm{h}}$, R. Nizzolo ${ }^{\mathrm{a}}$, A. Orlando ${ }^{\mathrm{c}}$,

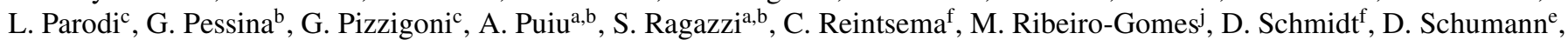

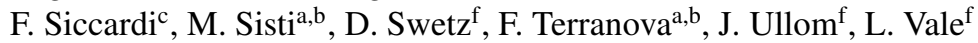

\author{
${ }^{a}$ Dipartimento di Fisica, Università di Milano-Bicocca, Milano, Italy \\ ${ }^{b}$ Istituto Nazionale di Fisica Nucleare (INFN), Sezione di Milano-Bicocca, Milano, Italy \\ ${ }^{c}$ Istituto Nazionale di Fisica Nucleare (INFN), Sezione di Genova, Genova, Italy \\ ${ }^{d}$ Jet Propulsion Laboratory, California Institute of Technology, Pasadena, California, USA \\ ${ }^{e}$ Paul Scherrer Institut (PSI), Villigen, Switzerland \\ ${ }^{f}$ National Institute of Standards and Technology (NIST), Boulder, Colorado, USA \\ ${ }^{g}$ Institut Laue-Langevin (ILL), Grenoble, France \\ ${ }^{h}$ Laboratori Nazionali del Gran Sasso (LNGS), INFN, Assergi (AQ), Italy \\ ${ }^{i}$ Istituto Nazionale di Fisica Nucleare (INFN), Sezione di Roma 1, Roma, Italy \\ ${ }^{j}$ Multidisciplinary Centre for Astrophysics (CENTRA-IST), University of Lisbon, Lisbon, Portugal
}

\begin{abstract}
HOLMES is a new experiment to directly measure the neutrino mass with a sensitivity as low as $0.4 \mathrm{eV}$. HOLMES will perform a calorimetric measurement of the energy released in the electron capture decay of ${ }^{163} \mathrm{Ho}$. HOLMES will deploy a large array of low temperature microcalorimeters with implanted ${ }^{163} \mathrm{Ho}$ nuclei. HOLMES baseline detector is an array of 1000 microcalorimeters each with an implanted ${ }^{163} \mathrm{Ho}$ activity of about $300 \mathrm{~Bq}$, an energy resolution FWHM of about $1 \mathrm{eV}$ at the spectrum end-point $(Q \approx 2.5 \mathrm{keV})$, and a time resolution of about $1 \mu \mathrm{s}$. Matching these performances requires a careful optimization of all components, from the microcalorimeters to the signal processing algorithms. We outline here the project technical challenges and the present status of the development.
\end{abstract}

Keywords: neutrino mass, Ho-163, electron capture, low temperature detectors

PACS: 23.40.Bw, 14.60.Pq, 07.20.Mc, 29.40.Vj

\section{Introduction}

Measuring the neutrino mass is one of the most compelling challenges of modern physics. Experiments studying oscillation phenomena have clearly shown that neutrinos have non vanishing mass and at least three neutrinos with different masses exist; yet the absolute values of these masses remain unknown. There are few methods from which neutrino mass can be assessed and nuclear beta decay spectrum end-point study is currently the one and only which can provide a model independent measurement of the absolute scale of neutrino mass. Within this framework the European Research Council has recently funded HOLMES, a new experiment to directly measure the neutrino mass [1]. HOLMES will perform a calorimetric measurement of the energy released in the electron capture decay of ${ }^{163} \mathrm{Ho}\left({ }^{163} \mathrm{Ho}+\mathrm{e}^{-} \rightarrow{ }^{163} \mathrm{Dy}^{*}+v_{\mathrm{e}}\right)$. This measurement was originally proposed in 1982 by A. De Rujula and M. Lusignoli [3], but only in the last decade the technological progress in detectors development allowed to design a sensitive experiment. In a calorimetric measurement the energy released in the decay process is entirely contained into the detector, except for the fraction taken away by the neutrino. The calorimetric approach eliminates both the problematic issues connected to the use of an external sources and the systematic uncertainties arising from decays to excited final states. The most suitable detectors for this type of measurement are low temperature thermal detectors, where all the energy released into an absorber is converted into a temperature increase that can be measured by a sensitive thermometer directly coupled with the absorber [2]. HOLMES will deploy a large array of low temperature microcalorimeters with implanted ${ }^{163} \mathrm{Ho}$ nuclei. The resulting mass sensitivity will be as low as $0.4 \mathrm{eV}$, depending on the $Q$ value of the transition which lacks a precise measurement, ranging from $2.3 \mathrm{keV}$ to $2.8 \mathrm{keV}$. HOLMES will be an important step forward in the direct neutrino mass measurement with a calorimetric approach as an alternative to spectrometry. It will also establish the potential of this approach to extend the sensitivity down to $0.1 \mathrm{eV}$ and lower.

${ }^{*}$ Corresponding author

Email address: angelo.nucciotti@mib.infn.it (A. Nucciotti) 


\section{HOLMES detectors R\&D}

\subsection{Baseline detector specifications}

In order to reach the sensitivity of $0.4 \mathrm{eV}$ it is necessary to collect a statistics of more than $10^{13}$ decays. To fulfill this task the best experimental configuration has been defined after Monte Carlo simulations [4]: in its optimal configuration HOLMES will collect about $3 \times 10^{13}$ decays with an instrumental energy resolution of about $1 \mathrm{eV}$ FWHM and a time resolution of about $1 \mu \mathrm{s}$. For a total measuring time of 3 years, this requires a total ${ }^{163} \mathrm{Ho}$ activity of about $3 \times 10^{5} \mathrm{~Bq}$. These Ho nuclei will be implanted in 1000 detectors.

\subsection{Signal processing}

HOLMES requires a very aggressive time resolution to minimize the number of unrecognized pile-up events. Pile-up happens when two decays in one detector are too close in time and are mistaken as a single one with an apparent energy equal to the sum of the two decays. This has a probability of $f_{p p}=\tau_{R} A$, where $\tau_{R}$ is the detector time resolution and $A$ is the ${ }^{163} \mathrm{Ho}$ activity in the detector. The energy spectrum of pile-up events is given by the self-convolution of the decay spectrum and extends up to $2 Q$, producing therefore a background impairing the ability to identify the neutrino mass effect at the decay spectrum end-point $Q$. Approximately $\tau_{R}$ is of the order of the detector rise time, but it actually depends on the detector signal bandwidth, on the high frequency signal-to-noise ratio, and on the amplitude of the two events to be resolved. A Montecarlo code has been developed to test algorithms for amplitude estimation and pile-up rejection on simulated digitized Transition Edge Sensor (TES) microcalorimeter signals in presence of noise and assess the achievable combined energy and time resolutions. We tested algorithms based on Optimal or Wiener Filtering and we found that a TES microcalorimeter designed to work at about $90 \mathrm{mK}$ with a heat capacity of about $0.5 \mathrm{pJ} / \mathrm{K}$, a rise time of about $5 \mu \mathrm{s}$, and a decay time of about $150 \mu \mathrm{s}$, could closely match the specifications. With a 12 bit digitization at $1 \mathrm{MS} / \mathrm{s}$, and a 512 sample record length, the algorithms we are testing give an energy and time resolution of about $2 \mathrm{eV}$ and $1.3 \mu \mathrm{s}$, respectively. Better algorithms are being tested to provide a more robust pile-up rejection and possibly relax both the detector and digitization specifications.

\subsection{Detector prototypes}

The detectors will be $\mathrm{Mo} / \mathrm{Cu}$ TES on $\mathrm{SiN}_{x}$ membrane with bismuth absorbers and will be fabricated in a two step process. The first steps will be carried out at the National Institute for Standard and Technology (NIST, Boulder, Co, USA) [5] where the devices will be fabricated up to the deposition of the bottom half of the absorber, i.e a $1.5 \mu \mathrm{m}$ bismuth layer. The devices will be then completed in the Genova INFN laboratory. Here, the most crucial step will be the deposition by means of the ion implanter of a thin (few $100 \AA$ ) layer of $\mathrm{Au}:{ }^{163} \mathrm{Ho}$. Then the absorber will be completed with a deposition of a second $1.5 \mu \mathrm{m}$ bismuth layer to fully encapsulate the ${ }^{163}$ Ho source. TES prototypes without the Au: ${ }^{163}$ Ho layer are being designed and fabricated according to the indications of our simulations. Preliminary tests gave devices with a heat capacity of about $0.5 \mathrm{pJ} / \mathrm{K}$, a rise time of about $10 \mu \mathrm{s}$, a decay time of about $130 \mu \mathrm{s}$, and a signal-to-noise corresponding to an energy resolution of about $2 \mathrm{eV}$. The rise time was slowed down on purpose by the $\mathrm{R} / \mathrm{L}$ constant of read-out circuit, but can be halved by proper tuning of the circuit.

\subsection{Detector read-out}

The signals from the 1000 microcalorimeters will be readout with a multiplexed system which is critical to preserve the performances of the individual detectors, especially in terms of available signal bandwidth, i.e. time resolution $\tau_{R}$. Microwave multiplexing ( $\mu \mathrm{MUX})$ is the most suitable system, since it gives a larger bandwidth for the same multiplexing factor (number of multiplexed detector signals). It is based on the use of rf-SQUIDs as input devices with flux ramp modulation [6]. The modulated rf-SQUID signals are read-out coupling the rf-SQUID to superconducting LC resonators in the $\mathrm{GHz}$ range and using the homodyne detection technique. By tuning the LC resonators at different frequencies it is straightforward to multiplex many RF carriers. The $\mu \mathrm{MUX}$ is suitable for a fully digital approach based on the Software Defined Radio (SDR) technique. The comb of frequency carriers is generated by digital synthesis in the $\mathrm{MHz}$ range and up-converted to the $\mathrm{GHz}$ range by $I Q$-mixing. The output signal is down-converted by $I Q$-mixing, sampled with a fast $\mathrm{A} / \mathrm{D}$ converter, and digital mixing techniques are used to recover the signals of each TES in the array (channelization) [7]. The multiplexing factor is set by the bandwidths of both the A/D converter and the TES signal. It can be shown that the multiplexing factor is approximately given by at most $0.02 f_{\mathrm{ADC}} \tau_{\text {rise }}$, where $f_{\mathrm{ADC}}$ is the $\mathrm{ADC}$ sampling frequency and $\tau_{\text {rise }}$ is the TES signal rise time. With the ADC boards currently available (550 MS/s, 12 bit) and with TESs similar to our prototypes, a multiplexing factor of about 50 is at reach. Increasing the multiplexing factor or decreasing the rise time requires faster $\mathrm{A} / \mathrm{D}$ converters.

\section{Conclusions}

We carried out preliminary tests with prototype devices without implanted ${ }^{163}$ Ho. Devices were designed according to the results of Montecarlo simulations and showed that presently HOLMES specifications can be matched within a factor 2 .

\section{Acknowledgments}

The HOLMES experiment is funded by the European Research Council under the European Union's Seventh Framework Programme (FP7/2007-2013)/ERC Grant Agreement no. 340321. We also acknowledge support from the NIST Innovations in Measurement Science program for the TES detector development. 


\section{References}

[1] B. Alpert et al., Eur. Phys. J. C 75, (2015) 112.

[2] C. Enss (Ed.), Cryogenic Particle Detection, Springer, Berlin, Heidelberg, 2005.

[3] A. De Rújula and M. Lusignoli, Phys. Lett. B 118, (1982) 429.

[4] A. Nucciotti, Eur. Phys. J. C 74, (2014) 11, arXiv:1405.5060.

[5] K. D. Irwin, G. C. Hilton, "Transition-edge sensors" in [2], pp. 63-149.

[6] O. Noroozian et al., Applied Phys. Lett. 103, (2013) 202602.

[7] S. McHugh et al., Rev. Sci. Instrum. 83, (2012) 044702. 